\title{
Kumaraswamy Distribution in Analyzing the Health-related Quality of Life and Effective Factors in Elderly Patients with Epilepsy
}

\author{
Soudabeh Hamedi-Shahraki ${ }^{1,2}$, Mohammad Reza Eshraghian ${ }^{2}$, Mir Saeed Yekaninejad ${ }^{2,{ }^{*}}$, Farshad \\ Amirkhizi $^{3}$, Aliakbar Rasekhi ${ }^{4}$ and Amir Pakpour ${ }^{5,6}$ \\ ${ }^{1}$ Department of Epidemiology and Biostatistics, School of Public Health, Zabol University of Medical Sciences, Zabol, Iran \\ ${ }^{2}$ Department of Epidemiology and Biostatistics, School of Public Health, Tehran University of Medical Sciences, Tehran, Iran \\ ${ }^{3}$ Department of Nutrition, School of Public Health, Zabol University of Medical Sciences, Zabol, Iran \\ ${ }^{4}$ Department of Biostatistics, Faculty of Medical Sciences, Tarbiat Modares University, Tehran, Iran \\ ${ }^{5}$ Social Determinants of Health Research Center, Qazvin University of Medical Sciences, Shahid Bahounar, Qazvin, Iran \\ ${ }^{6}$ Department of Nursing, School of Health and Welfare, Jönköping University, Jönköping, Sweden \\ "Corresponding author: Department of Epidemiology and Biostatistics, School of Public Health, Tehran University of Medical Sciences, Tehran, Iran. Email: \\ yekaninejad@yahoo.com
}

Received 2019 June 17; Revised 2019 July 27; Accepted 2019 August 07.

\begin{abstract}
Background: Epilepsy, which develops in the elderly, is recognized as a major health burden. Although health-related quality of life (HRQoL) is an essential element in the medical treatment of elderly patients with epilepsy, it is a question whether epilepsy and its treatment effectively influence the quality of life $(\mathrm{QoL})$ in the elderly.

Objectives: The current study aimed at evaluating the relationship between demographic and clinical aspects of epilepsy in HRQoL of elderly patients. Since HRQoL scores are bounded, the Kumaraswamy (Kum) regression model was used to analyze the data.

Methods: The current study was conducted on 766 elderly patients diagnosed with epilepsy taking at least one antiepileptic drug (AED) selected from six neurologic clinics in Iran. In addition to demographic information, the Liverpool seizure severity scale (LSSS), medication adherence report scale (MARS-5), and quality of life in epilepsy (QoLIE-31) questionnaire were completed for patients. Data were analyzed using multiple linear regression (MLR) and the Kum regression models.

Results: Most of the patients included in the study had focal (70.2\%) epilepsy. Mean duration of disease was $17.71 \pm 4.56$ years and the average number of seizures was $3.4 \pm 3.2$ episodes per month. The Kum regression model indicated that seizure frequency $(\beta=$ $-0.157, \mathrm{P}<0.0001)$ and LSSS score $(\beta=-0.003, \mathrm{P}=0.009)$ were significant and negative predictors of overall QoLEI-31 score; MARS-5 score was a positive predictor of overall QoLEI-31 score $(\beta=0.014, \mathrm{P}=0.002)$. However, disease duration and serum AED level had no significant effects on overall QoLEI-31 score.

Conclusions: The findings suggested that increased seizure frequency and severity were associated with lower QoL and medication adherence was directly associated with HRQoL. The Kum regression could be a suitable alternative to the methods currently used in the analysis of HRQoL data.
\end{abstract}

Keywords: Epilepsy, Health-related Quality of Life, Medication Adherence, Seizure, Kumaraswamy Regression

\section{Background}

Epilepsy is one of the most common disabling neurological disorders with a relatively high prevalence in the elderly after stroke and dementia (1). Epilepsy can markedly affect patients' health-related quality of life (HRQoL) and lead to a lower quality of life (QoL) by the impact on the mental and social aspects of the patients $(2,3)$. It is often necessary to assess the HRQoL of such patients in order to measure health status and evaluate medical care results. Validated tools to measure HRQoL can provide information about the disease and its impact on patients' lives, in a standardized, comparable and relatively objective manner
(4). Despite the importance of determining the treatment effects and individual characteristics on HRQoL, the appropriate analyzing method in this field is not completely understood yet, and various regression techniques (5-8) are proposed to analyze HRQoL data. Due to the simplicity of interpretation, multiple linear regression (MLR) is one of the techniques most widely used to study the effects of treatment on HRQoL and individual characteristics (8-12). However, due to some certain properties of HRQoL score, it is implausible that MLR is the most suitable method to analyze such data. Firstly, HRQoL outcomes have a double bounded format (e. g., 0 and 1); therefore, tend to be dis- 
tributed non-normally. Furthermore, the distribution of these outcomes is highly skewed. Due to these properties, they usually represent heteroscedasticity, where the variance is smaller near the extremes and asymmetry. Therefore, applying standard methods of analysis such as linear regression that assume normality and constant variances may not be appropriate for such data.

One of the distributions that may be suitable for modeling such data is Kumaraswamy (Kum) distribution. The Kum distribution was originally proposed by Kumaraswamy (13) for variables that are lower and upper bounded. The Kum distribution is highly flexible and its distribution can provide many shapes such as unimodal, increasing, decreasing, and uniform; therefore, it might be suitable for modeling variables observed on the finite interval such as HRQoL scores. Although MLR models are used in most studies that analyze HRQoL, it is a question whether the Kum distribution is better than MLR models to analyze such data. The current study primarily aimed at applying a specific class of regression models based on the Kum distribution (14) to address the features mentioned in the HRQoL data and comparing the Kum model and MLR performances.

\section{Objectives}

The dataset employed to examine the application of the Kum model corresponded to HRQoL data in elderly people with epilepsy. The current study focused on two main aspects: development of the regression model based on the Kum distribution for bounded data and its comparison with that of MLR method, particularly HRQoL, as response variables, and evaluating the relationship between HRQoL with demographic characteristics and clinical features of patients with epilepsy.

\section{Methods}

\subsection{Data Source}

The study was conducted on elderly patients diagnosed with epilepsy referred to six neurological clinics in Iran (two in Qazvin and four in Tehran) recruited by convenience sampling from March 2014 to December 2015. The diagnosis was based on the International League Against Epilepsy criteria (15). The six neurological clinics were asked to recruit patients based on the following inclusion criteria: age 65 years or above, receiving at least one antiepileptic drug (AED), and signing the consent form. The exclusion criteria were history of alcohol and/or drug abuse and history of cardiovascular disease, metabolic disorders such as diabetes, rheumatoid arthritis, hepatic diseases, malignant tumors, and kidney dysfunctions.
All eligible participants completed a questionnaire including items on demographic characteristics, clinical features, and the medication adherence at baseline. Medication adherence was evaluated through the medication adherence report scale (MARS-5) (16). It is a self-reporting questionnaire including five statements of non-adherent behaviors. The total score of MARS- 5 ranges 5 to 25, with higher scores indicating higher adherence.

Blood samples were collected from the median cubital vein prior to the next daily routine dose of a drug to measure serum AED levels after the six-month follow-up period. Serum AED levels were determined using a microparticle enzyme immunoassay kit (Abbott Axsym ${ }^{\circledR}$, Abbott Laboratories, Abbott Park, IL, USA) (17). Moreover, during the sixmonth follow-up, the patients were asked to complete the Liverpool seizure severity scale (LSSS) and the quality of life in epilepsy inventory-31 (QoLIE-31).

LSSS evaluates severity of seizure in patients with epilepsy. The LSSS scores range 0 to 100, with higher scores indicating the greater the perceived seizure severity (18). Furthermore, the QoLIE-31 has seven dimensions that measure different aspects of HRQoL (19). The total score of the QoLIE-31 varies from 0 to 1 and is calculated by weighting and summarizing its dimension scores; higher scores indicate a better HRQoL.

\subsection{Statistical Methods}

Continuous variables are expressed as mean \pm standard deviation (SD), and categorical variables are presented as absolute and relative frequencies. Multiple linear regression was used to investigate the relationship between the total score of QoLIE-31 with the demographic characteristics and clinical features of the participants. The presence of heteroscedasticity was detected by the Breusch-Pagan test available in Imtest package and based on the obtained results, MLR model showed heteroscedasticity.

HRQoL scores have a double bounded format; on the other hand, the linear regression does not consider the data being bounded; therefore, the Kum regression model was also applied to model the data. The Kum regression is a modeling approach, assuming that the dependent variable $\left(\mathrm{y}_{1}, \ldots, \mathrm{y}_{\mathrm{n}}\right)$ follows the Kum distribution with density function:

$$
\begin{aligned}
& f\left(y_{t} ; \omega_{t}, p\right)=\left(\frac{1}{b-a}\right) \frac{p \log (0.5)}{\log \left(1-\omega_{t}^{p}\right)} y_{t}^{p-1}\left(1-y_{t}^{p}\right)^{\frac{\log (0.5)}{\log \left(1-\omega_{t}^{p}\right)}}-1 \\
& \qquad 0<\mathrm{y}_{\mathrm{t}}<1 \\
& \text { where } 0<\omega_{\mathrm{t}}<1 \text {, is the median and } \mathrm{P}>0 \text { is the } \\
& \text { dispersion parameter. The Kum regression model is ob- } \\
& \text { tained by assuming that the median of } \mathrm{y}_{\mathrm{t}} \text { can be written as }
\end{aligned}
$$


$\operatorname{logit}\left(\omega_{t}\right)=\sum_{i=1}^{k} x_{t i} \beta_{i}$, where $\beta=\left(\beta_{1}, \ldots, \beta_{\mathrm{k}}\right)^{\mathrm{T}}$ is a vector of unknown regression parameters and $\mathrm{x}_{\mathrm{t} 1}, \ldots, \mathrm{x}_{\mathrm{tk}}$ are observations on k known and fixed covariates (14). The interpretation of the Kum regression coefficients is based on the odds ratio of the median score.

Finally, MLR and the Kum models were compared by the Akaike information criterion (AIC) and the Bayesian information criterion (BIC) and calculated as follows:

$A I C=-2 \ln L_{f i t}+2 k$

$B I C=-2 \ln L_{f i t}+2 k \ln N$

where $\operatorname{lnL} L_{\text {fit }}$ is the maximized log-likelihood, $\mathrm{k}$ is the number of parameters in the model, and $\mathrm{N}$ is the number of observations.

Data analysis was performed using the R statistical software version 3.5.1 (VGAM package), and the significance level was 0.05 .

\section{Results}

\subsection{Description of Dataset}

The study sample consisted of 766 elderly patients with epilepsy, aged above 65 years (mean $\pm S D=73.9 \pm 5.7$ ). The general and clinical characteristics of the participants are presented in Table1. Almost half of the participants were female (54.7\%), most of them were married (76\%), and about one-third of them were employed (36.0\%). Most of the patients had focal epilepsy (70.2\%), with an average disease duration of $17.71 \pm 4.56$ years and seizure frequency of 3.4 \pm 3.2 episodes per month. Nearly half of them (48.7\%) had a serum AED level lower than the therapeutic range (i e, nonadherent). Moreover, the mean overall score of QoLIE-31 in the study participants was $0.68 \pm 0.2$ (Table 1 ).

\subsection{Regression Models}

The results of MLR and the Kum regression models are presented in Table 2. According to MLR model, level of education $(\beta=0.004, \mathrm{P}=0.001)$ and MARS-5 score $(\beta=$ $0.003, \mathrm{P}<0.0001)$ were significant and positive predictors of the overall score of QoLIE-31. Conversely, QoLIE-31 score decreased by the increase of seizure frequency $(\beta=-0.043$, $\mathrm{P}<0.0001)$ and LSSS score $(\beta=-0.002, \mathrm{P}<0.0001)$. Similarly, based on the Kum regression model, level of education $(\beta=0.017, \mathrm{P}=0.003)$ and MARS-5 score $(\beta=0.014$, $\mathrm{P}=0.002$ ) were positively correlated with QoLEI-31 score, while seizure frequency $(\beta=-0.157, \mathrm{P}<0.0001)$ and LSSS score $(\beta=-0.003, \mathrm{P}=0.009)$ were inversely correlated with QoLEI-31 score. Moreover, gender $(\beta=-0.144, \mathrm{P}=0.026)$ and marital status $(\beta=0.137, \mathrm{P}=0.045)$ significantly associated with QoLEI-31 score in the Kum model, whilst in

\begin{tabular}{|c|c|}
\hline Variables & Values \\
\hline Age, $y$ & $73.9 \pm 5.7$ \\
\hline Education, y & $8.9 \pm 5.1$ \\
\hline \multicolumn{2}{|l|}{ Gender, No. (\%) } \\
\hline Male & $347(45.3)$ \\
\hline Female & $419(54.7)$ \\
\hline \multicolumn{2}{|l|}{ Marital status, No. (\%) } \\
\hline Married & $582(76.0)$ \\
\hline Unmarried & $184(24.0)$ \\
\hline \multicolumn{2}{|l|}{ Employment status, No. (\%) } \\
\hline Employed & $276(36.0)$ \\
\hline Unemployed & $490(64.0)$ \\
\hline \multicolumn{2}{|l|}{ Epilepsy type, No. (\%) } \\
\hline Generalized & $228(29.8)$ \\
\hline Focal & $538(70.2)$ \\
\hline Disease duration, $y$ & $17.71 \pm 4.56$ \\
\hline Seizure frequency & $3.4 \pm 3.2$ \\
\hline LSSS score & $54.9 \pm 23.5$ \\
\hline \multicolumn{2}{|l|}{ QOLIE-31 score } \\
\hline Seizure concerns & $0.77 \pm 0.35$ \\
\hline Cognitive function & $0.66 \pm 0.43$ \\
\hline Energy/fatigue & $0.59 \pm 0.19$ \\
\hline Emotional wellbeing & $0.61 \pm 0.19$ \\
\hline Social function & $0.78 \pm 0.22$ \\
\hline Medication efficacy & $0.79 \pm 0.24$ \\
\hline Overall quality of life & $0.62 \pm 0.20$ \\
\hline Overall score & $0.68 \pm 0.20$ \\
\hline MARS-5 score & $13.3 \pm 6.5$ \\
\hline \multicolumn{2}{|l|}{ Serum AED level, No. (\%) } \\
\hline Below therapeutic range & $373(48.7)$ \\
\hline Within therapeutic range & $295(38.5)$ \\
\hline Above therapeutic range & $98(12.8)$ \\
\hline
\end{tabular}

Abbreviations: AED, antiepileptic drug; LSSS, the Liverpool seizure severity scale; MARS-5, the 5-item medication adherence report scale; QoLIE-31, the quality of life in epilepsy inventory-31.

${ }^{\mathrm{a}}$ Values are expressed as mean $\pm \mathrm{SD}$ unless otherwise indicated.

MLR model these associations were insignificant. The comparison of AIC and BIC indicated that their values for the Kum model were smaller than that of MLR model (Table 2). Therefore, based on these criteria, the Kum model was preferable than MLR in fitting such data.

A common diagnostic tool for the MLR model is the normal Q-Q plot of residuals. Figure 1 shows the normal Q- 


\begin{tabular}{|c|c|c|c|c|}
\hline \multirow[t]{2}{*}{ Covariate } & \multicolumn{2}{|c|}{ MLR Model } & \multicolumn{2}{|c|}{ Kum Regression } \\
\hline & $\beta(\mathbf{S E})$ & PValue $^{\mathrm{a}}$ & $\beta(\mathrm{SE})$ & P Value $^{\mathrm{a}}$ \\
\hline Intercept & $0.662(0.044)$ & $<0.0001$ & $0.768(0.222)$ & $<0.0001$ \\
\hline Gender & & 0.345 & & 0.026 \\
\hline Marital status & & 0.575 & & 0.045 \\
\hline Married & $0.008(0.014)$ & & $0.137(0.068)$ & \\
\hline Education, y & $0.004(0.001)$ & 0.001 & $0.017(0.006)$ & 0.003 \\
\hline Employment status & & 0.080 & & 0.085 \\
\hline Employed & $0.025(0.014)$ & & $0.120(0.070)$ & \\
\hline Serum AED level & $-0.004(0.008)$ & 0.657 & $-0.003(0.042)$ & 0.940 \\
\hline Seizure frequency & $-0.043(0.002)$ & $<0.0001$ & $-0.157(0.007)$ & $<0.0001$ \\
\hline LSSS score & $-0.002(0.001)$ & $<0.0001$ & $-0.003(0.001)$ & 0.009 \\
\hline MARS-5 score & $0.003(0.001)$ & $<0.0001$ & $0.014(0.005)$ & 0.002 \\
\hline Epilepsy type & & 0.911 & & 0.245 \\
\hline Generalized & $0.001(0.011)$ & & $0.067(0.058)$ & \\
\hline \multicolumn{5}{|c|}{ Goodness-of-Fit Statistics } \\
\hline AIC & -710.1 & & -758.8 & \\
\hline BIC & -654.5 & & -703.1 & \\
\hline $\mathbf{R}^{2}$ & 0.465 & & 0.404 & \\
\hline
\end{tabular}

Abbreviations: AED, antiepileptic drug; Kum, the Kumaraswamy; LSSS, the Liverpool seizure severity scale; MARS-5, the 5-item medication adherence report scale; MLR, multiple linear regression; QoLIE-31, the quality of life in epilepsy inventory-31; SE, standard error.

${ }^{\text {a }}$ Significance level $<0.05$.

Q plot for QoLIE-31 scores. As shown in Figure 1, the points at the two ends of the plots in the MLR model are not close to a straight line; therefore, the residuals are not normally distributed.

\section{Discussion}

The current study was conducted on elderly patients with focal and generalized epilepsy in which the association of patients' and diseases' characteristics with HRQoL was evaluated using both MLR and the Kum models. The study results indicated a significant reduction in HRQoL with increasing the frequency and severity of seizure among elderlies with epilepsy. Numerous studies examined the association of seizure frequency and severity with QoL. Adebayo et al. using the same seizure severity tool as that of the current study reported a significant decrease in HRQoL of adult patients with epilepsy, following the increase in seizure severity (20). In another study by Tedrus et al. greater seizure frequencies were the main factors influencing QoL as evaluated by QoLIE-31 in patients with epilepsy (8). Furthermore, seizure frequency is reported as a negative predictor of QoL in adults with epilepsy $(3,21)$. These results indicated the importance of encouraging patients to record the frequency and severity of their seizure episodes and share the information with their healthcare provider to improve the HRQoL.

Poor adherence to medication was previously reported as an important predictor of HRQoL in patients with epilepsy (22-24). In the current study, medication adherence was also directly associated with HRQoL among the patients. In accordance with the current study results, Ettinger et al. demonstrated that HRQoL directly affects the QoL (22). Moreover, in a study by Martins et al. scores of adherence to treatment strongly correlated with all QoLIE31 domains indicating higher HRQoL in patients with good medication adherence (25). Based on these findings, nonadherence to medication may lead to uncontrolled seizure with substantial deleterious effects on HRQoL. Therefore, the response to treatment may be an important issue to improve HRQoL among the elderly with epilepsy.

In the current study, a significant and positive associa- 

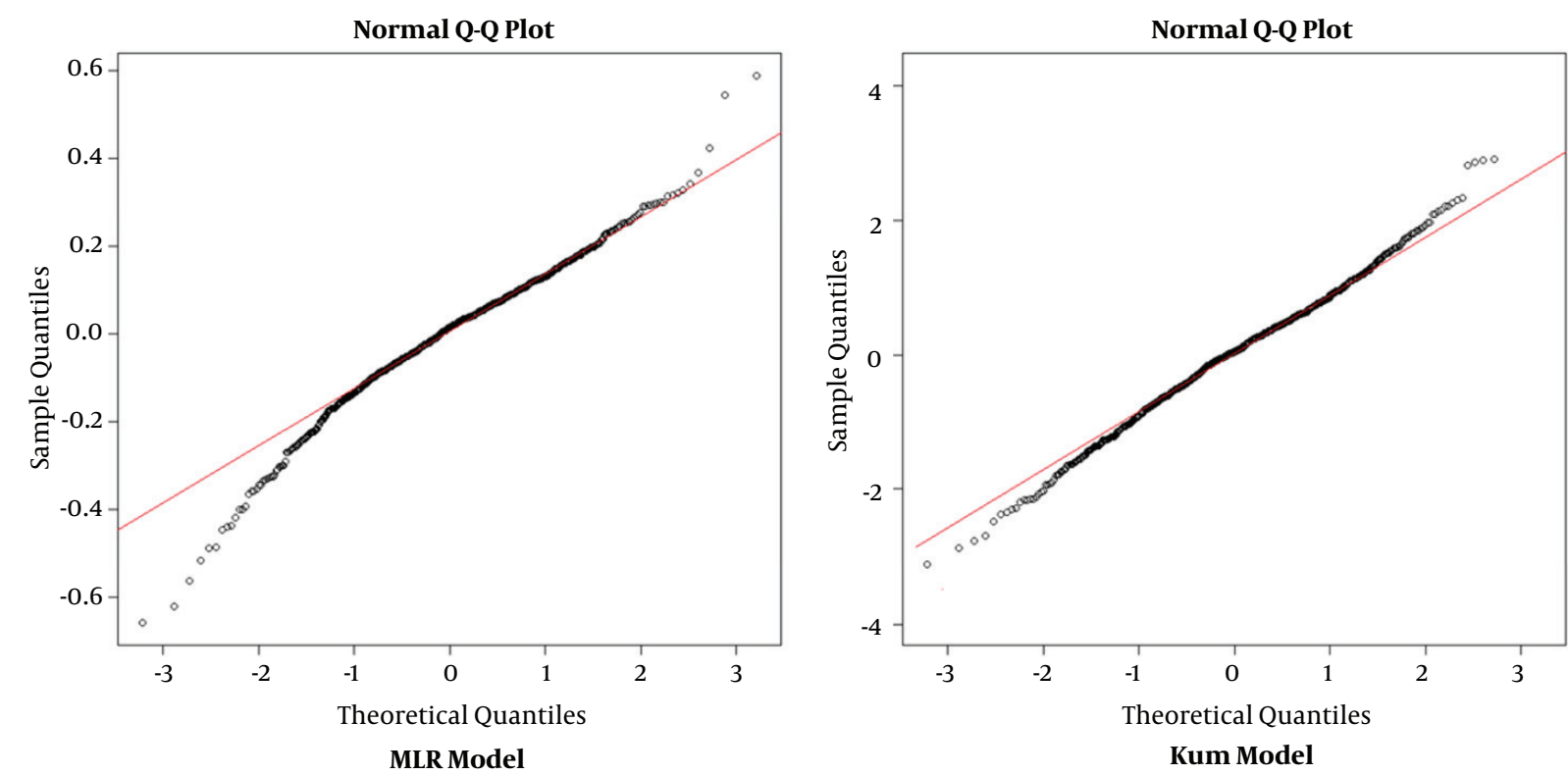

Figure 1. Normal probability plot; MLR, multiple linear regression; Kum, Kumaraswamy

tion was observed between education level of patients and overall score of QoLIE-31. Numerous studies examined the relationship between education levels and HRQoL in patients with epilepsy. For example, in a study by Ridsdale et al. education level was linearly associated with the higher QoL in patients with drug-resistant epilepsy (23). In addition, other studies illustrated a direct association between education level and QoL in patients with epilepsy (3, 26, 27). This may indicate that patients with higher level of education are aware of different aspects of the disease and may improve their QoL through appropriate treatment and seizure control. Certainly, further studies are required to obtain more conclusive results.

Based on the Kum model, other factors that influenced patients' overall HRQoL score in the current study included gender and marital status. According to the findings of the current study, being female and unmarried were significant predictors of lower QoL in the Kum model; however, these associations were not significant in the MLR model. Similarly, Nabukenya et al. in their study reported that being female and unmarried were negative predictors of HRQoL in patients with epilepsy (3). Other studies also reported that gender influenced HRQoL in patients with epilepsy; female patients had significantly lower HRQoL than males, which was in agreement with the current study results $(21,27)$. Based on these findings, healthcare providers should recognize that gender might be a factor when evaluating HRQoL in the elderly patients with epilepsy. Moreover, being married affords a social and psy- chological support and, hence, reduces the negative impact of lower scores of these aspects on overall HRQoL.

The limitations of the present study should be considered in the interpretation of the study findings. Firstly, the participants included in the study were recruited from six urban clinics in Qazvin and Tehran where the QoL might be higher compared to the population living in rural areas. Therefore, the findings may not be generalizable to all patients with epilepsy in Iran. Secondly, due to participants' conditions, sometimes they could not understand the questions asked, necessitating the use of a caretaker to interpret the questions for them and sometimes clarify on the patients' responses. There is a possibility that the caretakers did not interpret the questions correctly, which might yield a wrong response. However, all the caretakers were oriented on the meaning of the different questions and their comprehension of the questions was evaluated. Finally, authors could not assess mental, emotional, psychological, and socioeconomic conditions in the study subjects. The current study also had several strengths including a relatively large sample size, an adequate followup period, and its longitudinal design.

In conclusion, the present study revealed that female gender, seizure frequency, and seizure severity were negatively correlated with HRQoL, while higher education level, being married, and medication adherence were positively correlated. Therefore, all strategies and health policies to improve HRQoL in elderly patients with epilepsy should be based on improving medication adherence and seizure- 
related factors.

\section{Acknowledgments}

The authors extend their sincerest thanks to all the patients who served as samples of the study.

\section{Footnotes}

Authors' Contribution: Study concept and design: Amir Pakpour, Mir Saeed Yekaninejad and Mohammad Reza Eshraghian; analysis and interpretation of data: Soudabeh Hamedi-Shahraki and Aliakbar Rasekhi; drafting of the manuscript: Soudabeh Hamedi-Shahraki; critical revision of the manuscript for important intellectual content: Farshad Amirkhizi and Mir Saeed Yekaninejad.

Conflict of Interests: The authors declared no conflict of interest.

\section{Ethical Considerations: Ethical code} IR.TUMS.SPH.REC.1396.2907.

Funding/Support: There was no financial support for the study.

\section{References}

1. Johnston A, Smith PE. Epilepsy in the elderly. Expert Rev Neurother 2010;10(12):1899-910. doi: 10.1586/ern.10.170. [PubMed: 21384700].

2. Edefonti V, Bravi F, Turner K, Beghi E, Canevini MP, Ferraroni M, et al. Health-related quality of life in adults with epilepsy: The effect of age, age at onset and duration of epilepsy in a multicentre Italian study. BMC Neurol. 2011;11:33. doi: 10.1186/1471-2377-11-33. [PubMed: 21392391]. [PubMed Central: PMC3062600].

3. Nabukenya AM, Matovu JK, Wabwire-Mangen F, Wanyenze RK, Makumbi F. Health-related quality of life in epilepsy patients receiving anti-epileptic drugs at National Referral Hospitals in Uganda: A cross-sectional study. Health Qual Life Outcomes. 2014;12:49. doi: 10.1186/1477-7525-12-49. [PubMed: 24725904]. [PubMed Central: PMC3999846].

4. Goldsmith SB. The status of health status indicators. Health Serv Rep. 1972;87(3):212-20. doi: 10.2307/4594470. [PubMed: 4554009]. [PubMed Central: PMC1615939]

5. Walters SJ, Campbell MJ, Lall R. Design and analysis of trials with quality of life as an outcome: A practical guide. J Biopharm Stat. 2001;11(3):155-76. doi: 10.1081/BIP-100107655. [PubMed: 11725929].

6. Lall R, Campbell MJ, Walters SJ, Morgan K. A review of ordinal regression models applied on health-related quality of life assessments. Stat Methods Med Res. 2002;11(1):49-67. doi: 10.1191/0962280202sm271ra. [PubMed: 11923993].

7. Mesbah M,Cole BF, Lee MLT. Statistical methods for quality of life studies: design, measurements and analysis. Springer Science \& Business Media; 2013.

8. Tedrus GM, Fonseca LC, Carvalho RM. Epilepsy and quality of life: Socio-demographic and clinical aspects, and psychiatric comorbidity. Arq Neuropsiquiatr. 2013;71(6):385-91. doi: 10.1590/0004282X20130044. [PubMed: 23828532].

9. Austin PC. A comparison of methods for analyzing health-related quality-of-life measures. Value Health. 2002;5(4):329-37. doi: 10.1046/j.1524-4733.2002.54128.x. [PubMed: 12102695].
10. Wee HL, Cheung YB, Loke WC, Tan CB, Chow MH, Li SC, et al The association of body mass index with health-related quality of life: An exploratory study in a multiethnic Asian population. Value Health. 2008;11 Suppl 1:S105-14. doi: 10.1111/j.1524-4733.2008.00374.x. [PubMed: 18387053].

11. Derakhshanpour F, Vakili MA, Farsinia M, Mirkarimi K. Depression and quality of life in patients with type 2 diabetes. Iran Red Crescent Med J. 2015;17(5). e27676. doi: 10.5812/ircmj.17(5)2015.27676.

12. Pellise F, Vila-Casademunt A, Ferrer M, Domingo-Sabat M, Bago J, Perez-Grueso FJ, et al. Impact on health related quality of life of adult spinal deformity (ASD) compared with other chronic conditions. Eur Spine J. 2015;24(1):3-11. doi: 10.1007/s00586-014-3542-1. [PubMed: 25218732].

13. Kumaraswamy P. A generalized probability density function for double-bounded random processes. J Hydrol. 1980;46(1-2):79-88. doi: 10.1016/0022-1694(80)90036-0.

14. Bonat WH, Ribeiro Jr PJ, Zeviani WM. Regression models with responses on the unit interval: specification, estimation and comparison. Biom Braz J. 2012;30(4):415-31.

15. Berg AT, Berkovic SF, Brodie MJ, Buchhalter J, Cross JH, van Emde Boas W, et al. Revised terminology and concepts for organization of seizures and epilepsies: Report of the ILAE Commission on Classification and Terminology, 2005-2009. Epilepsia. 2010;51(4):676-85. doi 10.1111/j.1528-1167.2010.02522.x. [PubMed: 20196795].

16. Lin CY, Yaseri M, Pakpour AH, Malm D, Brostrom A, Fridlund B, et al. Can a multifaceted intervention including motivational interviewing improve medication adherence, quality of life, and mortality rates in older patients undergoing coronary artery bypass surgery? A multicenter, randomized controlled trial with 18-month followup. Drugs Aging. 2017;34(2):143-56. doi: 10.1007/s40266-016-0429-3. [PubMed: 28004259]

17. Affolter N, Krahenbuhl S, Schlienger RG. Appropriateness of serum level determinations of antiepileptic drugs. Swiss Med Wkly. 2003;133(43-44):591-7. [PubMed: 14745654].

18. Scott-Lennox J, Bryant-Comstock L, Lennox R, Baker GA. Reliability, validity and responsiveness of a revised scoring system for the Liverpool Seizure Severity Scale. Epilepsy Res. 2001;44(1):53-63. doi 10.1016/s0920-1211(01)00186-3. [PubMed: 11255073].

19. Cramer JA, Van Hammee G, N. Study Group. Maintenance of improvement in health-related quality of life during long-term treatment with levetiracetam. Epilepsy Behav. 2003;4(2):118-23. doi:10.1016/S15255050(03)00004-0. [PubMed: 12697135].

20. Adebayo PB, Akinyemi RO, Ogun SA, Ogunniyi A. Seizure severity and health-related quality of life of adult Nigerian patients with epilepsy. Acta Neurol Scand. 2014;129(2):102-8. doi: 10.1111/ane.12146. [PubMed: 23682560]

21. Chen HF, Tsai YF, Hsi MS, Chen JC. Factors affecting quality of life in adults with epilepsy in Taiwan: A cross-sectional, correlational study. Epilepsy Behav. 2016;58:26-32. doi: 10.1016/j.yebeh.2016.02.019. [PubMed: 27002941].

22. Ettinger AB, Good MB, Manjunath R, Edward Faught R, Bancroft T. The relationship of depression to antiepileptic drug adherence and quality of life in epilepsy. Epilepsy Behav. 2014;36:138-43. doi: 10.1016/j.yebeh.2014.05.011. [PubMed: 24926942].

23. Ridsdale L, Wojewodka G, Robinson E, Landau S, Noble A, Taylor $S$, et al. Characteristics associated with quality of life among people with drug-resistant epilepsy. J Neurol. 2017;264(6):1174-84. doi: 10.1007/s00415-017-8512-1. [PubMed: 28550480]. [PubMed Central: PMC5486549].

24. Hamedi-Shahraki S, Eshraghian MR, Yekaninejad MS, Nikoobakht M, Rasekhi A, Chen $\mathrm{H}$, et al. Health-related quality of life and medication adherence in elderly patients with epilepsy. Neurol NeurochirPol.2019;53(2):123-30. doi:10.5603/PJNNS.a2019.0008. [PubMed: 30807640]. 
25. Martins HH, Alonso NB, Guilhoto LMFF, Guaranha MSB, Yacubian EMT. Adherence to treatment in patients with juvenile myoclonic epilepsy: Correlation with quality of life and adverse effects of medication. J Epilepsy Clin Neurophysiol. 2009;15(4):192-6. doi: 10.1590/s167626492009000400010.

26. Jacoby A, Baker GA. Quality-of-life trajectories in epilepsy: A review of the literature. Epilepsy Behav. 2008,12(4):557-71. doi: 10.1016/j.yebeh.2007.11.013. [PubMed: 18158270].

27. Kovats D, Csaszar N, Haller J, Juhos V, Sallay V, Bekes J, et al. Factors affecting quality of life in Hungarian adults with epilepsy: A comparison of four psychiatric instruments. Epilepsy Behav. 2017;74:45-58. doi: 10.1016/j.yebeh.2017.04.035. [PubMed: 28686907]. 